\title{
ELECTROSTATIC CURVED ELECTRODE ACTUATORS
}

\author{
Rob Legtenberg, Erwin Berenschot, Miko Elwenspoek and Jan Fluitman \\ MESA Research Institute, University of Twente, P.O. Box 217, 7500 AE Enschede, \\ The Netherlands
}

\begin{abstract}
In this paper, the design, fabrication, and first experimental results of electrostatic curved electrode actuators are addressed. The actuator design is based upon the deformation of a movable micromechanical structure by electrostatic forces using a fixed curved electrode. When a voltage is applied, an electrostatic force is created that will deform the free structure along the outline of the fixed curved electrode. To investigate the basic phenomena of this type of actuators, research has been focused on cantilever beam structures. For the shape of the curved electrode simple polynomials, normalized to the maximal deflection, have been used. A theory is presented, describing the static behaviour of this type of actuators. Devices are fabricated from polysilicon by surface micromachining techniques. Displacements of several tens of micrometers and forces of several $\mu \mathrm{N}$ can be generated for cantilever beams with a length of several hundred micrometers.
\end{abstract}

\section{Introduction}

Electrostatic actuation is very attractive for microelectro-mechanical systems because of good scaling properties to small dimensions, high energy densities and its relative ease of fabrication. However, electrostatic actuators which are able to generate relatively large displacements and large forces are difficult to design as a result of a geometric discrepancy. Large force actuators (e.g. parallel plate structures) require small gaps and a displacement in the direction of the major field lines, thus implying small displacements. In contrast, large displacement actuators (e.g. comb drive structures) require displacements perpendicular to the major field lines, leading to small forces. By employing curved structures large displacement actuators can be realized [1-4].

In this paper the design and performance is presented of an actuator design that is based upon the deformation of a movable micromechanical structure by means of electrostatic forces using a fixed curved electrode.

\section{Design}

The design of curved electrode actuators includes a movable electrode structure, e.g. a cantilever beam, and a mechanically fixed curved counter electrode as shown in Fig. 1. The gap distance between both electrodes is small near the clamped edge of the beam and slowly increases towards the point of maximum displacement of the beam. When a voltage is applied across the gap, an electrostatic force is created that will deform the free structure along the outline of the fixed curved electrode.

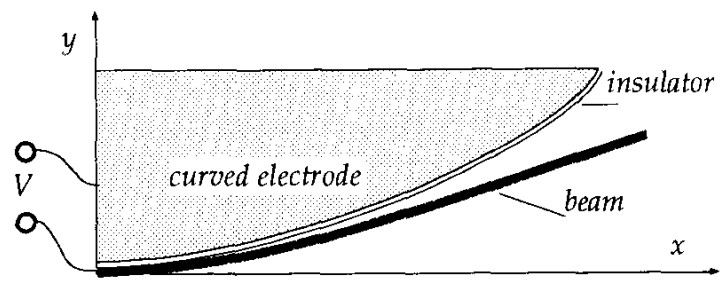

Fig.I Schematic of the curved electrode actuator

The displacement is parallel to the wafer surface. To prevent short circuiting between the beam and the curved electrodes, electrical insulation is required e.g. by applying a dielectric layer between the structures or by using stand off bumper structures that prevent physical contact of the electrodes.

Our initial work has been focussed on cantilever beam structures in order to study the basic behaviour of this type of actuators without too complex mathematics. However similar structures can be fabricated using microbridges or membranes.

For the shape of the curved electrode simple polynomials have been used that have been normalized to a maximum tip deflection of the cantilever beam. As will be shown in the next section the performance of these actuators is depending on the electrode curvature and can become unstable after the so-called pull-in voltage.

\section{Theory}

\section{Unloaded static behaviour:}

Because the gap spacing is small with respect to the electrode length it is assumed that the electric field lines cross the gap only in the y-direction, i.e. a parallel plate approximation.

When a d.c. polarization voltage is applied between the capacitor electrodes, an electrostatic force is developed that is inversely proportional to the square of the gap spacing. This makes the force dependent on the deflection, leading to non-linear behaviour. The static deflection $w_{(x)}$ of a prismatic beam with a transverse pressure $q_{(x, V)}$ can be 
described by the following non-linear differential equation of equilibrium:

$$
E I \frac{d^{4} w(x)}{d x^{4}}=q(x, V)=\frac{1}{2} \frac{\varepsilon_{0} h V^{2}}{\left[\frac{d}{\varepsilon_{r}}+s(x)^{-n}(x)\right]^{2}}
$$

where $q(x, V)$ denotes the static electrostatic force per unit beam length as a function of the position $x$ and the drive voltage $V, E I$ is the bending stifness, $\mathrm{d}$ is the thickness of the insulator, $\varepsilon_{0}$ the dielectric constant of the spacing medium, $h$ the width of the beam, $\varepsilon_{r}$ is the dielectric constant of the insulator and $s_{(x)}$ the shape of the electrode as a function of the position $x$. An analytical closed-form solution of the above equation cannot be found and numerical solutions or a simplified model based on the method of total potential energy using small deflection theory has to be applied e.g. the Rayleigh-Ritz method, were an approximate solution to the differential equation is constructed in the form of admissible trial functions $[5,6]$. The total potential energy, denoted by $\Pi$, can be expressed as:

$$
\Pi=U_{b}+V_{e l}
$$

where $U_{b}$ and $V_{e l}$ are the strain energy term of bending and the potential energy of the electrostatic force $q_{(x, V)}$ given by:

$U_{b}=\frac{1}{2} \int_{0}^{L} E I\left[\frac{d^{2} w(x)}{d x^{2}}\right]^{2} d x$

and

$V_{e l}=-\frac{1}{2} \int_{0}^{L} \frac{\varepsilon_{0} h V^{2}}{\frac{d}{\varepsilon_{r}}+s(x)-w(x)} d x$

For the admissible trial function of the deflection profile of the cantilever beam, the deflection profile of a uniformly loaded cantilever beam has been used:

$$
\tilde{w}(x)=c g(x)=c x^{2}\left(6 L^{2}-4 L x+x^{2}\right)
$$

where $c$ is a constant that has to be determined. The following expression has been used for the shape of the curved electrodes:

$$
s(x)=\delta_{\max }\left[\frac{x}{L}\right]^{n}
$$

where $\delta_{\max }$ is the maximum gap distance of the curved electrode and $n$ is the order of the curve, $n \geq$ 0 .

The system is in equilibrium when the first variation of the potential energy with respect to the constant $c$ equals zero. By solving this equation the unknown $c$ can be found giving the approximate deflection profile.

Whether this equilibrium is stable or unstable is determined by the second variation of the potential energy with respect to $c$. At the transition from a stable to an unstable equilibrium the first and the second derivative of the potential energy with respect to $c$ are zero. Solving these equations simultaneously yields the pull-in voltage $V_{P I}$ of the cantilever and an implicit expression for the constant $c_{P I}$ at pull-in:

$V_{P I}^{2}=\frac{E I}{\varepsilon_{0} h} \frac{\int_{0}^{L}\left[\frac{d^{2} g(x)}{d x^{2}}\right]^{2} d x}{\int_{0}^{L} \frac{g(x)^{2}}{\left(\frac{d}{\varepsilon_{r}}+s_{(x)}-c_{P I} g(x)\right)^{3}} d x}$

$$
\int_{0}^{L} \frac{{ }^{c} P I g(x)^{2} d x}{\left(\frac{d}{\varepsilon_{r}}+s(x)^{-c} P I g(x)\right)^{3}}=\int_{0}^{L} \frac{g(x) d x}{\left(\frac{d}{\varepsilon_{r}}+s(x)^{-c} P I g(x)\right)^{2}}
$$

The calculated pull-in voltages for different polynomial orders obtained by this energy method are listed in table 1.

Equations (7) and (8) do not give much insight in the effect of the parameters. Increasing the polynomial order $n$ of the electrode curve, decreases the pull-in voltage while remaining about the same maximum displacement. By using curved electrodes the pull-in voltage can be lowered significantly resulting in large amplitude motion at low driving voltages compared to the parallel plate structure $(n=0)$. However at voltages higher than the pull-in voltage the displacement cannot be controlled hecause of the unstable nature. 


\begin{tabular}{|c||c|c|}
\hline$n$ & $V_{p i}[V]$ & $c_{p i}\left[* 10^{7} \mathrm{~m}^{-3}\right]$ \\
\hline \hline 0 & 141.7 & 7.63 \\
\hline 0.5 & 115.2 & 7.66 \\
\hline 1.0 & 86.2 & 7.56 \\
\hline 1.5 & 59.5 & 6.88 \\
\hline 2.0 & 40.0 & 5.54 \\
\hline
\end{tabular}

Table 1 Pull-in voltage of polysilicon ( $E=150 \mathrm{GPa})$ cantilever beams $\left(h^{*} t^{*} L=5 * 2 * 500 \mu \mathrm{m}\right)$ with a minimum gap spacing $d$ of $2 \mu \mathrm{m}$ and a maximum deflection $\delta_{\text {max }}$ of $30 \mu \mathrm{m}$. The relative dielectric constant of the insulator $\varepsilon_{r}=$ I.

The pull-in voltage is strongly dependent on the beam thickness and the initial gap spacing at the clamped edge of the beam.

An interesting effect that can be noticed is that the maximum tip displacement at pull-in is independent of the beam length. The tip displacement at pull-in calculated by our model is roughly about one third of the maximum gap spacing $\delta_{\max }$ which is equal to the stable to unstable deflection boundary for a lumped parallel-plate spring model [7]. However decreasing the beam length increases the pull-in voltage. A larger dielectric constant of the insulating layer between the electrodes will reduce the pull-in voltage.

The pull-in voltage keeps decreasing with increasing polynomial order. However for polynomial orders higher than two it was found that the deflection profile of the beam becomes constrained by the geometry of the curved electrode before the pull-in voltage is reached and the model has to be adjusted. When the deflection of the beam becomes constrained by the curved electrode, this adjusted model predicts a stable behaviour of the beam untill it is completely deformed along the outline of the curved electrode. However this still has to be experimentally confirmed and is under research.

\section{Force generation and external loading:}

In this section the force generation of a curved electrode will be modelled. An external force $P$ acting on the tip of the beam will bend the beam in addition to the electrostatic forces, in that part where the beam is not in contact with the curved electrode (see Fig. 2).

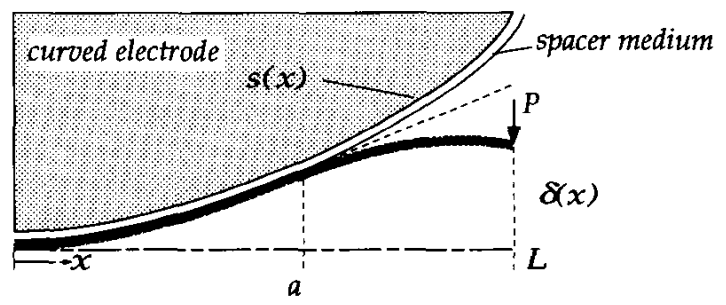

Fig. 2 Sketch of the model with external loading force $P$.
It is assumed that the beam will be partly in contact with the curved electrode and will partly be free standing as sketched above, and is clamped at point $a$. The problem will have a variable boundary condition with respect to the free standing length of the beam. Therefore the system has to be divided in two regions. From the clamped edge of the beam to point $a$, the beam is in physical contact with the curved electrode and the deflection profile $w_{(x)}$ will be equal to the shape of the electrode $s_{(x)}$ and the distance between the electrodes is equal to $d$, the thickness of the insulator. Beyond point $a$, the beam is free and deflected by electrostatic forces. The expressions for the strain energy of bending and the potential energy of the electrostatic force now are:

$$
\begin{aligned}
U_{b}=\frac{1}{2} \int_{0}^{a} E I & {\left[\frac{d^{2} s(x)}{d x^{2}}\right]^{2} d x } \\
& +\frac{1}{2} \int_{a}^{L} E I\left[\frac{d^{2} w(x)}{d x^{2}}\right]^{2} d x
\end{aligned}
$$

and

$$
\begin{aligned}
V_{e l}=-\frac{1}{2} \int_{0}^{a} \frac{\varepsilon_{r} \varepsilon_{0} h V^{2}}{d} d x \\
-\frac{1}{2} \int_{a}^{L} \frac{\varepsilon_{0} h V^{2}}{\frac{d}{\varepsilon_{r}}+s(x)-w(x)} d x
\end{aligned}
$$

An additional term has to be added to the total potential energy, given in expression (2). This term is the work from the external force acting on the tip of the beam, given by:

$$
V_{P}=P w(L)
$$

The admissible trial function of the deflection profile of the cantilever beam will now be dependent on the contact distance $a$ and needs to be adjusted by bending of force $P$ :

$$
\begin{gathered}
\tilde{w(x)=s(x)} \quad \text { for } 0<x \leq a \\
\tilde{w(x)}=c(x-a)^{2}\left[6(L-a)^{2}-4(L-a)(x-a)+(x-a)^{2}\right] \\
-\frac{P(x-a)^{2}[3(L-a)-(x-a)]}{6 E I}+ \\
{[s(x)]_{x=a}+\left[\frac{d s}{d x}\right]_{x=a}(x-a)}
\end{gathered}
$$




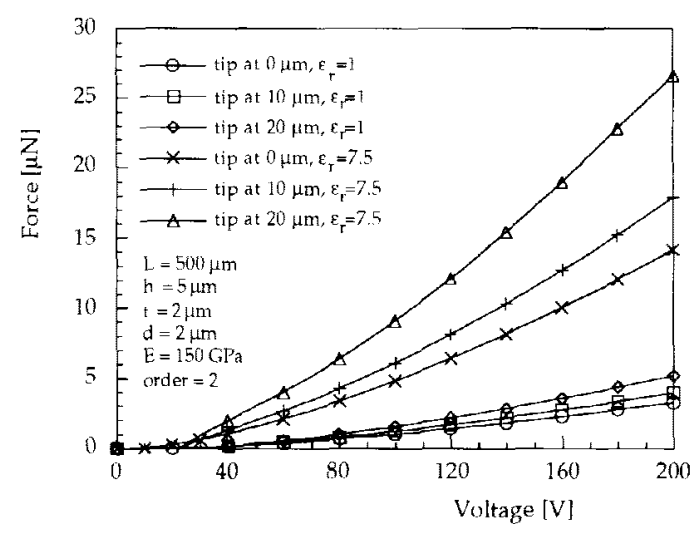

Fig. 3 Theoretical force generation of a quadratic order electrade curvature as a function of driving voltage. The tip deflection is fixed at 0,10 and $20 \mu \mathrm{m}$. Also the effect of the relative dielectric constant of the insulating layer is shown.

The system, now, is in equilibrium when the first variation of the potential energy with respect to the contact distance $a$ and the constant $c$ equals zero. Solving both equations simultaneously, by numerical iteration, gives the values of $a$ and $c$ at a certain driving voltage. The force generated by the actuator is a function of the displacement of the tip. The external force to keep the tip fixed at a certain position is shown in fig. 3 for different tip positions. In equilibrium this equals the force generated by the actuator. Also the effect of the dielectric constant of the insulating layer between the electrodes is shown. The actuator dimensions are also shown in fig. 3.

\section{Fabrication}

A one mask fabrication process has hecn developed where electrical isolation between the electrodes is accomplished by polysilicon bumper structures.

The fabrication starts with a (100) p-type 3 " silicon wafer. First step is wet thermal oxidation at $1150{ }^{\circ} \mathrm{C}$ to obtain a $2 \mu \mathrm{m}$ thick $\mathrm{SiO}_{2}$ layer. Next a $5 \mu \mathrm{m}$ thick polysilicon layer is grown by LPCVD at a temperature of $590{ }^{\circ} \mathrm{C}$, a pressure of $250 \mathrm{mT}$ orr and a silane flow of $50 \mathrm{sccm}$. This polysilicon layer is heavily doped with boron by means of deposition of a BSG layer and indiffusion at $1150^{\circ} \mathrm{C}$ for 3 hours. This yields a squared resistance of about $4.5 \Omega$ and also results in a small residual strain of the polysilicon layer. After boron indiffusion the BSG layer is stripped in a buffered HF-solution. A 0.6 $\mu \mathrm{m}$ thick PECVD silicon oxide layer is grown that serves as an etch mask for the polysilicon. After patterning the silicon oxide by RIE using $\mathrm{CHF}_{3}$ gas, the polysilicon is anisotropically etched using a $\mathrm{SF}_{6}$.
$\mathrm{O}_{2}, \mathrm{CHF}_{3}$ gas mixture, After a cleaning step the sacrificial layer is etched for 30 minutes in a buffered HF solution. This only frees the thin beam but keeps the other structures still attached to the substrate. Drying is done by means of a special freeze drying method to prevent stiction of free structures to the substrate [8]. Finally a $1 \mu \mathrm{m}$ thick aluminium layer is evaporated for backside contact. The final result is shown in fig. 4 and 5 .

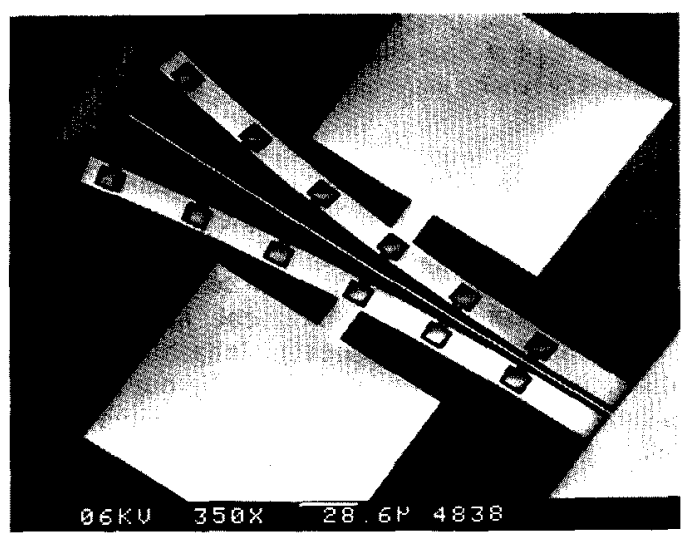

Fig. 4 SEM photograph of curved electrode actuator with stand off bumpers.

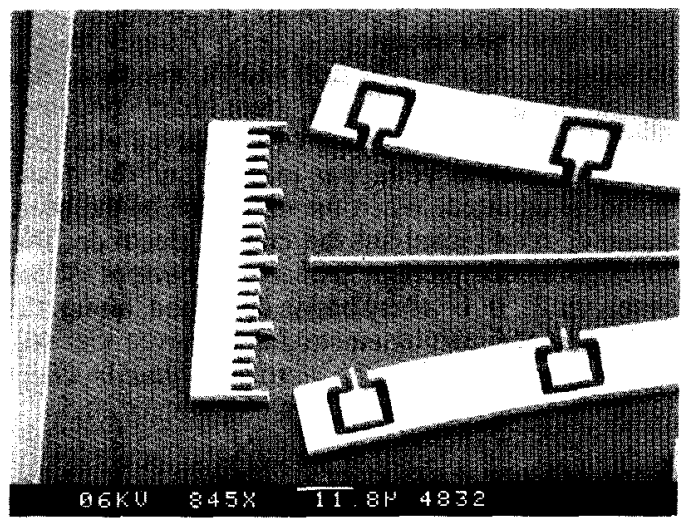

Fig. 5 Close up of the free standing actuator tip and the siand off bumper structures.

\section{Results and Discussion}

Experimental Set-up:

The tip deflection as a function of the applied driving voltage has been measured for different electrode curves using an experimental set-up consisting of a probe station with a microscope and a digital voltage supply. The beam electrode and the substrate are connected to ground potential while the curved electrode is connected to a positive voltage. Deflections and dimensions have been measured using a micrometer eyepiece. 


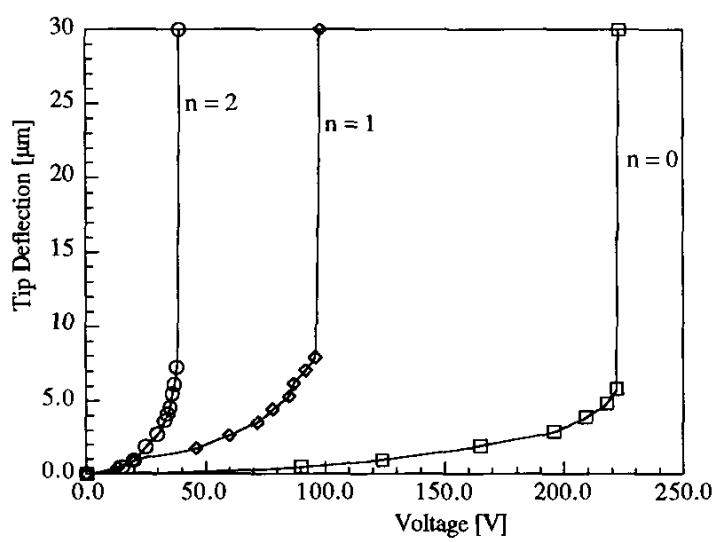

Fig.6 Measured tip deflection versus driving voltage for instable electrode profiles. The polynomial orders are 2, 1 and $D$.

\begin{tabular}{|c||c|c|}
\hline \multirow{2}{*}{ order } & \multicolumn{2}{c|}{ Pull-in Voltage [V] } \\
\cline { 2 - 3 } & \multicolumn{2}{|c|}{ Cantilever } \\
\cline { 2 - 3 } & Exp. & Theory \\
\hline 0 & 223 & 112.1 \\
\hline 1.0 & 98 & 74.4 \\
\hline 2.0 & 40 & 36.9 \\
\hline
\end{tabular}

Table 2 Pull-in voltage of polysilicon ( $E=150 \mathrm{GPa})$ cantilever beams using bumper structures with a maximum deflection $\delta_{\text {max }}$ of $30 \mu \mathrm{m}$. The thickness of the poly layer is $4.6 \mu \mathrm{m}$ (width of the beam), the thickness of the sacr. layer is $1.6 \mu \mathrm{m}$. The thickness of the beams are 1.6,1.7 and $1.8 \mu \mathrm{m}$ and the initial gap is $2.2,3.2$ and $3.3 \mu \mathrm{m}$ for the $0, I$ and 2 order polynomials respectively.

Pull-in voltage of instable bumper designs:

To determine the pull-in voltage, the driving voltage was slowly increased untill the beam deflection became unstable. The results are shown fig. 6 and the pull-in voltages are listed in table 2 .

The calculated pull-in voltages are smaller than the measured values. The difference is increasing with decreasing order of the curvature. This effect can be explained by three dimensional electrostatic field effects. Electrostatic fringing fields will increase the electrostatic forces and will result in lower pull-in voltages than calculated by our simple parallel plate approximation. However the presence of the groundplane gives rise to an unbalanced electrostatic field distribution [9]. This effect reduces the electrostatic forces, especially for large gaps, and induces a levitation of the surface micromachined structure. These effects can be modelled by a 3D coupled electromechanical solver like CoSolve-EM [10]. The pull-in voltage will increase by the presence of the groundplane. The effect is stronger for increasing gap distances. This makes it difficult to give accurate quantitative predictions of the pull-in voltage by a simple onedimensional model and makes 3D simulations necessary. Furthermore the pull-in voltage is very sensitive to the thickness of the beam and the initial gap spacing. For instance a change in beam thickness of $0.1 \mu \mathrm{m}$ results in a change of 10 percent in the pull-in voltage of a second order polynomial design. The qualitative behaviour of the model and the experiments are in agreement with eachother.

The behaviour of the actuators shows a hysteresis effect for increasing and decreasing voltages. This has been studied in more detail for the second order polynomial electrodes and the experimental results are shown in fig. 7. The origin of the hysteresis comes from a difference in the electrostatic field distribution between a free and a pulled-in beam. Once the beam is pulled-in, the electrostatic forces strongly increase as a result of the decreased gap spacing and a large decrease in the driving voltage is needed before the beam releases.

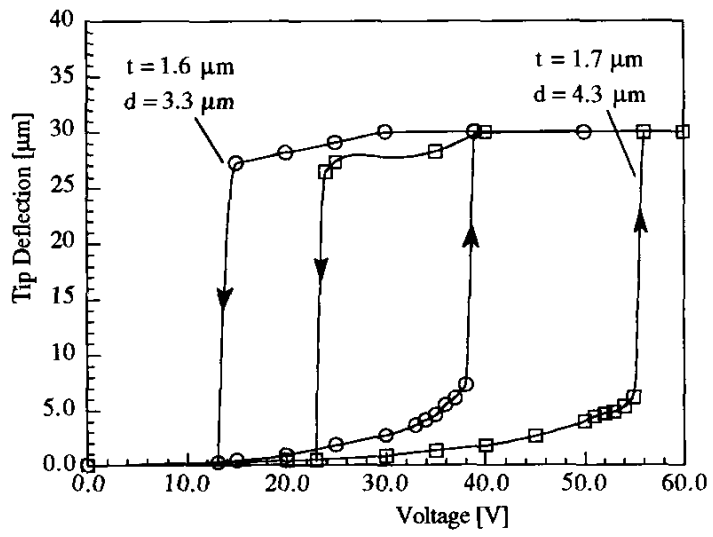

Fig. 7 Measurement of the hysteresis effect for second order polynomial designs. Beam length and width are respectively $500 \mu \mathrm{m}$ and $4.6 \mu \mathrm{m}$. Beam thickness and initial gap spacing are 1.6, 3.3 $\mu \mathrm{m}$ and $1.7,4.3 \mu \mathrm{m}$ respectively.

Constrained bumper designs:

When the polynomial order becomes larger than 2 the deflection of the beam becomes constrained by the geometry of the curved electrode as discussed earlier. In case of a design with stand off bumper structures this will give a stepwise instable behaviour as the beam reaches an instable point from bumper to bumper. The measured deflection behaviour of a third and fourth order polynomial curvature design with bumper structures at every 50 
$\mu \mathrm{m}$ is shown in fig. 8 for beams with a length of 500 $\mu \mathrm{m}$.

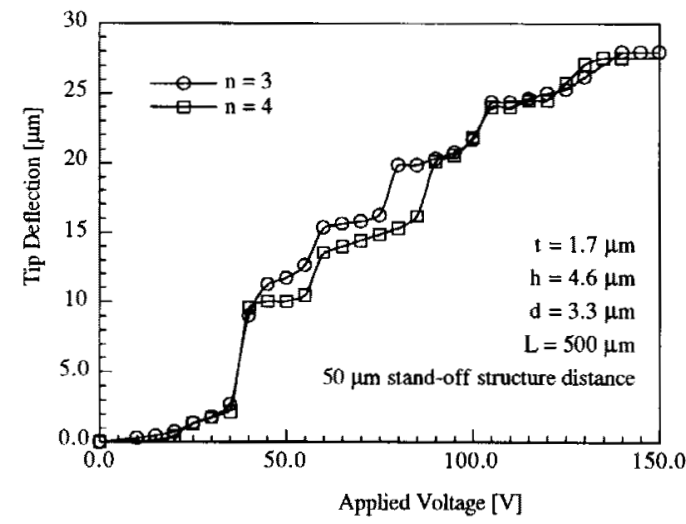

Fig. 8 Experimental results of curved electrodes with stand off bumper structures where the beam deflection becomes constrained by the electrode geometry (bumpers). The polynomial orders are 3 and 4.

These results indicate that constrained designs can lead to a completely stable actuator performance when the beam can smoothly zip along the curved electrode. This may be realized by increasing the amount of bumper structures or by a insulating layer between the electrodes.

\section{Conclusions}

An electrostatic actuator design has been presented where a deformable mechanical structure is bend around a fixed curved clectrode by means of electrostatic forces. For the shape of the curved electrode simple polynomials have been used. A theory based upon energy methods is presented to describe the static behaviour of the actuator. When the beam deflection is not constrained by the curved electrode geometry, instable behaviour occurs at a certain pull-in voltage and a hysteresis exist to release the structure after pull-in. For designs with an order higher than two it was found that the beam deflection can become constrained by the curved electrode geometry before pull-in. Curved electrode actuators have been fabricated from polysilicon by surface micromachining techniques using a onemask process where electric insulation has been realized by stand off bumper structures between the movable beam and the fixed electrode. Measurements of non-constrained beam deflections show that the qualitative behaviour is in agreement with theory but that the pull-in voltages are higher than theoretically predicted. This can be explained by 3-D electrostatic effects. Constrained designs employing bumper structures show a stepwise behaviour as a result of a number of stable positions at the bumper positions. Deflections of several tens of micrometers are obtained and theoretical force generation is on the order of several $\mu \mathrm{N}$.

\section{Acknowledgements}

The authors thank Bert Geyselears for helpfull discussions, Harrie Tilmans for carefully reading the manuscripts and Nico van Dellen for his help with the measurements. Also they like to thank Bert Otter for making the SEM photographs. This research is sponsored by the Dutch Technology Foundation (STW).

\section{References}

[1] K. Sato and M. Shikida, Electrostatic film actuator with large vertical displacement, Proc. IEEE Micro Electro Mechanical Systems, Travemunde, Germany, February 4-7, 1992, pp. 1-5.

[2] J. Branebjerg and P. Gravesen, A new electrostatic actuator providing improved stroke length and force, Proc. IEEE Micro Electro Mechanical Systems, Travemunde, Germany, February 4-7, 1992, pp. 6-11.

[3] M. Elwenspoek, L. Smith and B. Hök, Active joints for microrobot limbs, J.Micrmech. Microeng., 2 (1992), pp. 221-223.

[4] M. Yamaguchi, S. Kawamura, K Minami and M Esashi, Distributed electrostatic micro actuator, Proc. IEEE Micro Electro Mechanical Systems, Fort Lauderdale, Florida, U.S.A., February 7 10,1993 , pp. $18-23$.

[5] I.H. Shames and C.L. Dym, Energy and finite element methods in structural mechanics, McGraw-Hill, New York, 1985.

[6] H. Tilmans and R. Legtenberg, Electrostatically driven vacuum encapsulated polysilicon resonators-Part II. S\&A, A45, 1994, pp. 67-84.

[7] P. Osterberg, H. Yie, X. Cai, J. White and S. Senturia, Self consistent simulation and modelling of electrostatically deformed diaphragms, Proc. IEEE Micro Electro Mechanical Systems, Oiso, Japan., February 25 28, 1994, pp. 28-32.

[8] R. Legtenberg and H.A.C. Tilmans, Electrostatically driven vacuum encapsulated polysilicon resonators-Part I, S\&A, A45, 1994 , pp. 57-66.

[9] W.C. Tang, M.G. Lim and R.T. Howe, Electrostatic comb drive levitation and control method, J. of MEMS, Vol. 1, No. 4, 1992, pp. 170-174

[10] J.R. Gilbert, R. Legtenberg and S.D Senturia, 3D coupled electro-mechanics for MEMS: Applications of CoSolve-EM, Proc. IEEE Micro Electro Mechanical Systems, Amsterdam, The Netherlands, January 30 February 2, 1995. 